1. Bauman Л, Bauernfeind RA, Hoff JV, Strasberg B, Swiryn S, Rosen KM. Tor sades de pointes due to quinidine: observations in 31 patients. Am Heart $J 1984$; $107: 425-430$.

2. Roden DM, Woosley RL, Primm RK. Incidence and clinical features of the quinidine-associated long QT syndrome: implications for patient care. Am Heart J 1986; 111:1088-1093.

3. Coplen SE, Antman EM, Berlin JA, Hewitt P, Chalmers TC. Efficacy and safety of quinidine therapy for maintenance of sinus rhythm after cardioversion: a metaanalysis of randomized control trials. Circulation 1990;82:1106-1116.

4. The Stroke Prevention in Atrial Fibrillation (SPAF) Investigators. Stroke prevention in atrial fibrillation study: final results. Circulation 1991;84:527-539.
5. Bazett HC. An analysis of the time-relations of the electrocardiogram. Hear 1920;7:353.

6. Lopez AJ, Harold JG, Rosenthal MC, Oseran DS, Schapira JN, Peter T. QT prolongation and torsades de pointes after administration of trimethoprim-sulfamethoxazole. Am J Cardiol 1987:59:376-377.

7. Gohn DC. Simmons TW. Polymorphic ventricular tachycardia (torsades de pointes) associated with the use of probucol. N Engl J Med 1992;326:1435-1436. 8. Strasberg B, Scharovsky S, Erdberg A, Duffy CE, Lam W, Swiryn S, Agmon J, Rosen KM. Procainamide-induced polymorphous ventricular tachycardia. Am J Cardiol 1981;47:1309-1314

\title{
Placement of Electrode Catheters into the Coronary Sinus During Electrophysiology Procedures Using a Femoral Vein Approach
}

Emile G. Daoud, MD, Mark Niebauer, MD, Omar Bakr, MD, John Jentzer, MD, K. Ching Man, DO, Brian D. Williamson, MD, John D. Hummel, MD, S. Adam Strickberger, MD, and Fred Morady, MD

E lectrograms recorded from within the coronary sinus provide useful diagnostic and mapping data in patients undergoing electrophysiologic procedures for management of paroxysmal supraventricular tachycardia or Wolff-Parkinson-White syndrome. ${ }^{1,2}$ Conventionally, an electrode catheter is inserted into the coronary sinus using a subclavian, internal jugular, or brachial vein approach. ${ }^{3,4}$ However, it is also possible to catheterize the coronary sinus using a femoral vein approach, thereby eliminating the need for any venous access other than the femoral vein. The purpose of this study was to prospectively assess the feasibility of placing an electrode catheter into the coronary sinus using a femoral vein approach.

The subjects of this study were 105 consecutive patients who underwent an electrophysiologic procedure at the University of Michigan Medical Center. In 85 patients, a femoral vein approach was used to catheterize the coronary sinus. In 20 patients who were undergoing evaluation of a recently implanted internal cardioverter! defibrillator, the electrophysiologic procedure was performed with a single electrode catheter using an internal jugular or subclavian vein approach. Patients who underwent the femoral vein approach had a mean age $( \pm S D)$ of $45 \pm 18$ years and 60 of 85 patients $(70 \%)$ had no structural heart disease. Patients who underwent an internal jugular or subclavian vein approach had a mean age of $58 \pm 15$ years and only 1 of 20 patients (5\%) did not have structural heart disease.

Among the 85 patients who underwent a femoral vein approach, 3 sheaths were inserted into the right femoral vein and $36 \mathrm{Fr}$ or $7 \mathrm{Fr}$ electrode catheters were positioned in the right atrium. His bundle position, and right ventricle. At least 1 of these catheters had a deflectable tip (Mansfield, Watertown, Massachusetts). After baseline electrophysiologic measurements were obtained, the deflectable tip catheter was directed into the coronary sinus under fluoroscopic guidance using a $60^{\circ}$ left anterior oblique view (Figure 1). The amount of time re-

From the Division of Cardiology, Department of Medicine, University of Michigan Medical Center, 1500 East Medical Center Drive, Ann Arbor, Michigan 48109-0022. Manuscript received October 19, 1993; revised manuscript received and accepted December 14, 1993 quired to maneuver the catheter from its prior position into the lateral coronary sinus was measured with a stopwatch. Among the 20 patients who underwent an internal jugular or subclavian vein approach, the deflectable tip catheter was inserted through a sheath placed into the left subclavian or right internal jugular vein and maneuvered into the coronary sinus. The time required to cannulate the vein and catheterize the coronary sinus was measured. With both approaches, a second operator attempted to catheterize the coronary sinus if the first operator was not successful within 15 minutes.

Continuous variables are expressed as mean $\pm S D$ and were compared using the Student's $\mathrm{t}$ test. A $p$ value $<0.05$ was considered significant.

Using the femoral vein approach, the coronary sinus was successfully catheterized in 84 of 85 patients (99\%) in a mean of $1.2 \pm 2.3$ minutes. The catheterization was achieved by the first operator in $92 \%$ of patients and by

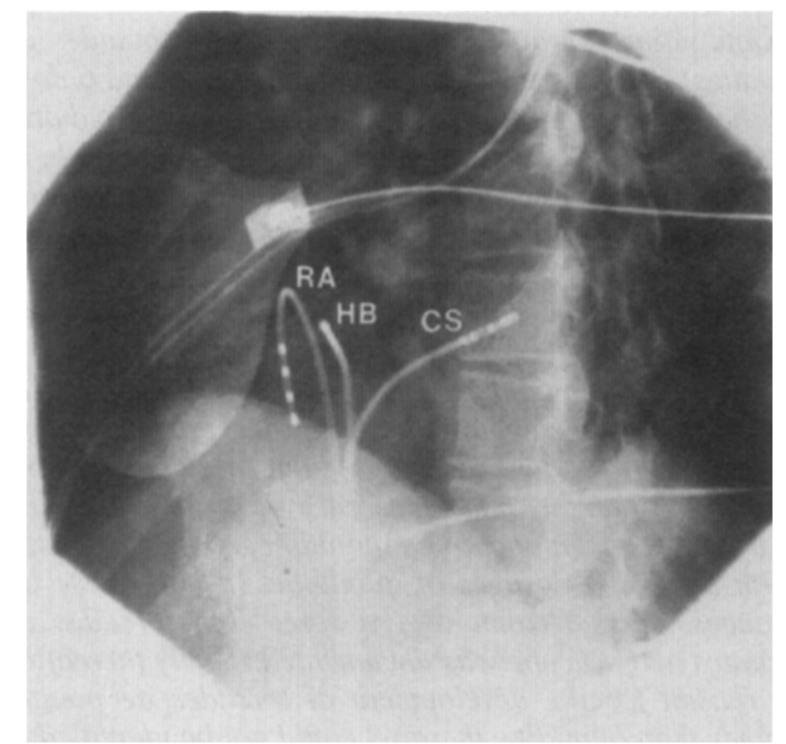

FGURE 1. Fluoroscopic $60^{\circ}$ left anterior oblique view of a deflectable catheter in the coronary sinus (CS). Catheters in the His bundle (HB) position and right atrium (RA) are also shown. With an appropriate degree of tip deflection, the anticlpated region of the coronary sinus ostium was probed with the catheter until the coronary sinus was entered. 
a second operator in $7 \%$. The coronary sinus could not be successfully catheterized in an obese patient with dilated cardiomyopathy and 4-chamber cardiac enlargement. Catheterization of the coronary sinus was successful in each of the 20 patients who underwent the internal jugular or subclavian approach, and the mean time required to achieve catheterization was $12.5 \pm 10$ minutes, which was significantly longer than with the femoral vein approach $(p<0.001)$. There were no complications in any of the patients in this study.

The results of this study demonstrate that catheterization of the coronary sinus during an electrophysiologic procedure can almost always be accomplished in a brief period of time using a deflectable tip catheter inserted into a femoral vein. The femoral vein approach saves time because it precludes the need for cannulation of the subclavian, internal jugular, or brachial vein. The possible complications associated with an internal jugular or subclavian vein approach are avoided, including pneumothorax, hemothorax, thoracic duct injury, laceration of a carotid or cervical artery, brachial plexus injury, tracheal perforation, air embolism, and aortic puncture. 5 The femoral vein approach also has a cost advantage over the internal jugular and subclavian approaches. Because it is quicker, the laboratory charge for the electrophysiologic procedure is lower than when additional time is spent cannulating a vein other than the femoral vein. Because one of the three catheters inserted for baseline measurements is used, the expense of a fourth venous access kit and electrode catheter is spared. In addition, a postprocedure chest $\mathrm{x}$-ray is not necessary to rule out a pneumothorax when the femoral vein approach is used. The cost savings associated with use of the femoral vein approach instead of a subclavian or internal jugular approach may often total several hundred dollars per patient.

The femoral vein approach used in this study has 2 limitations. First, it requires the use of a deflectable tip catheter, which typically is $\$ 200$ to $\$ 300$ more expensive than a standard electrode catheter. Second, with the femoral vein approach used in the present study, the total number of pacing and recording sites is limited to 3 . However, because the coronary sinus catheter can be used for atrial pacing, and because the right atrial electrogram is rarely required for diagnostic purposes, we have found the combination of the coronary sinus, His bundle electrogram, and right ventricular apex positions to be adequate in virtually all patients undergoing an electrophysiologic procedure in whom coronary sinus electrograms are needed for a diagnostic or mapping purpose.

1. Calkins H, Sousa J, El-Atassi R, Kosenheck S, Buitleir M, Kou KH, Kadish AH, Langberg JJ, Morady F. Diagnosis and cure of the Wolff-Parkinson-White syndrome or paroxysmal supraventricular tachycardias during a single electrophysiologic test. N Engl J Med 1991;324:1612-1618.

2. Szabo TS, Klein GJ, Guiraudon GM, Yee R, Sharma AD. Localization of accessory pathways in the Wolff-Parkinson-White syndrome. PACE 1989:12 1691-1705.

3. Michelson EL, Medina RP. Introduction to clinical electrophysiologic studies. Cardiovasc Clin 1985;16:1-36.

4. Hammill SC, Sugrue DD, Gersh BJ, Porter CJ, Osborn MJ, Wood DL, Holmes DR. Clinical intracardiac electrophysiologic testing: technique, diagnostic indications, and therapeutic uses. Mayo Clin Proc 1986;61:478-503.

5. Mitchell SE, Clark RA. Complications of central venous catheterization. A.IR 1979;133:467-476

6. Orebaugh SL. Venous air embolism: clinical and experimental considerations Crit Care Med 1992;20:1169-1177.

\title{
The Effects of Adenosine on Idiopathic Ventricular Tachycardia
}

\author{
Kheng-Siang Ng, MBBS, MRCP, Ming-Shien Wen, MD, San-Jou Yeh, MD, Fun-Chung Lin, MD, and Delon Wu, MD
}

$\mathrm{V}$ entricular tachycardia (VT) in the absence of structural heart disease is an uncommon but well-recognized entity. Two clinical forms have often been observed: (1) verapamil-sensitive VT characterized by right bundle branch block (RBBB) morphology with a superior frontal plane axis, and (2) catecholamine-sensitive VT characterized by left bundle branch block (LBBB) morphology with an inferior frontal plane axis. The former typically originates from the inferoapical portion of the left ventricle, can be initiated by programmed stimulation, and terminated with intravenous verapamil, ${ }^{1,2}$ whereas the latter typically originates from the right ventricular outflow tract, requires adrencrgic

From the 2nd Section of Cardiology, Department of Medicine, Chang Gung Memorial Hospital, Chang Gung Medical College, 199 Tung IIwa North Road, Taipei, Taiwan. This study was supported in part by Grant DOH83-HR-205 from the National Health Institute, Taipei, and Grants NSC82-O412-B182-022 and NSC82-O115-B182-081 from the National Science Council of the Republic of China, Taipei, Taiwan. Manuscript received November 1, 1993; revised manuscript received and accepted December 20, 1993. stimulation for initiation, and can be terminated by both $\beta$-blocker and verapamil administration. ${ }^{3}$ Attempts have been made to identify the VT mechanism by response to programmed stimulation and pharmacologic manipulation (isoproterenol and verapamil), but the distinction between triggered automaticity and reentry remains potentially difficult. ${ }^{4}$ Lerman et $\mathrm{al}^{5}$ recently provided strong evidence for cyclic adenosine- $3^{\prime} 5^{\prime}$-monophosphate-mediated triggered automaticity as the mechanism in patients in whom adenosine reliably terminated the tachycardia. The drug did not terminate tachycardia in patients with reentrant VT. These observations suggest that adenosine may therefore be used as a diagnostic tool to distinguish the mechanism of VT. We report the outcome of the effect of adenosine triphosphate on our series of patients with idiopathic VT.

Drug testing with adenosine triphosphate was performed in 28 patients $(19$ males and 9 females, mean age $31 \pm 15$ years, range 9 to 61) with electrocardiographic documentation of spontaneous idiopathic VT. All patients had normal results on clinical examination, chest $x$-ray, and 2-dimensional Doppler echocardiogra- 